\title{
BMJ Open Patient-centred care, health behaviours and cardiovascular risk factor levels in people with recently diagnosed type 2 diabetes: 5-year follow-up of the ADDITION-Plus trial cohort
}

\author{
Hajira Dambha-Miller, ${ }^{1,2}$ Andrew J M Cooper, ${ }^{2}$ Rebecca K Simmons, ${ }^{2}$ \\ Ann Louise Kinmonth, ${ }^{1}$ Simon J Griffin ${ }^{1,2}$
}

To cite: Dambha-Miller $\mathrm{H}$, Cooper AJM, Simmons RK, et al. Patient-centred care, health behaviours and cardiovascular risk factor levels in people with recently diagnosed type 2 diabetes: 5-year follow-up of the ADDITION-Plus trial cohort. BMJ Open 2016;6:e008931. doi:10.1136/bmjopen-2015008931

- Prepublication history for this paper is available online. To view these files please visit the journal online (http://dx.doi.org/10.1136/ bmjopen-2015-008931).

Received 29 May 2015 Revised 21 October 2015 Accepted 26 October 2015

CrossMark

For numbered affiliations see end of article.

Correspondence to Dr Hajira Dambha-Miller; hd322@cam.ac.uk

\section{ABSTRACT}

Objective: To examine the association between the experience of patient-centred care (PCC), health behaviours and cardiovascular disease (CVD) risk factor levels among people with type 2 diabetes.

Design: Population-based prospective cohort study. Setting: 34 general practices in East Anglia, UK, delivering organised diabetes care.

Participants: 478 patients recently diagnosed with type 2 diabetes aged between 40 and 69 years enrolled in the ADDITION-Plus trial.

Main outcome measures: Self-reported and objectively measured health behaviours (diet, physical activity, smoking status), CVD risk factor levels (blood pressure, lipid levels, glycated haemoglobin, body mass index, waist circumference) and modelled 10year CVD risk.

Results: Better experiences of PCC early in the course of living with diabetes were not associated with meaningful differences in self-reported physical activity levels including total activity energy expenditure ( $\beta$-coefficient: $0.080 \mathrm{MET}$ h/day $(95 \% \mathrm{Cl} 0.017$ to $0.143 ; p=0.01))$, moderate-to-vigorous physical activity ( $\beta$-coefficient: $5.328 \mathrm{~min} /$ day ( $95 \% \mathrm{Cl} 0.796$ to 9.859 ; $\mathrm{p}=0.01)$ ) and reduced sedentary time ( $\beta$-coefficient: $-1.633 \mathrm{~min} /$ day $(95 \% \mathrm{Cl}-2.897$ to $-0.368 ; \mathrm{p}=0.01)$ ). PCC was not associated with clinically meaningful differences in levels of high-density lipoprotein cholesterol ( $\beta$-coefficient: $0.002 \mathrm{mmol} / \mathrm{L}(95 \% \mathrm{Cl} 0.001$ to $0.004 ; p=0.03)$ ), systolic blood pressure ( $\beta$-coefficient: $-0.561 \mathrm{~mm} \mathrm{Hg}(95 \% \mathrm{Cl}-0.653$ to $-0.468 ; p=0.01))$ or diastolic blood pressure ( $\beta$-coefficient: $-0.565 \mathrm{~mm} \mathrm{Hg}(95 \% \mathrm{Cl}-0.654$ to $-0.476 ; p=0.01))$. Over an extended follow-up of 5 years, we observed no clear evidence that PCC was associated with self-reported, clinical or biochemical outcomes, except for waist circumference ( $\beta$ coefficient: $0.085 \mathrm{~cm}(95 \% \mathrm{Cl} 0.015$ to 0.155 ; $\mathrm{p}=0.02)$ ).

Conclusions: We found little evidence that experience of PCC early in the course of diabetes was associated with clinically important changes in health-related behaviours or CVD risk factors.

\section{Strengths and limitations of this study}

- Our study is the first to use objective measures of health behaviours to examine the impact of patient-centred care in recently diagnosed type 2 diabetes.

- The study duration is 5 years with high rates of follow-up.

- We included a large number of general practitioner (GP) surgeries that reflects the average UK GP list size, number of doctors/nurses and diabetes prevalence.

- Patient-centred care was only measured at a single time point.

- The majority of our participants were Caucasian males with high levels of education and employment, thereby limiting the generalisability of our findings.

Trial registration number: ISRCTN99175498; Postresults.

\section{INTRODUCTION}

Type 2 diabetes is a common condition mostly managed in general practice. Despite current lifestyle and medication treatments, patients with diabetes still have high rates of cardiovascular disease (CVD) morbidity and mortality. ${ }^{1}$ Patient-centred care (PCC) is considered the cornerstone of UK general practice and may play an important role in the management of CVD risk factor levels. ${ }^{2}$ By understanding individual health beliefs, considering patient preferences and developing mutual management plans, general practitioners (GPs) may be able to positively influence health behaviours such as diet, physical activity, smoking and alcohol intake, each of 
which are known to influence CVD risk factor levels. ${ }^{3}$ This potentially effective and cost-effective role for GPs in influencing patient health behaviours has recently been emphasised in national and international health policy. ${ }^{4}{ }^{5}$ The majority of supporting evidence comes from observational data reporting inverse associations between PCC and CVD risk factor levels. ${ }^{7}$ Trial findings have been more variable with some studies reporting no effect from interventions promoting PCC, while others report reduced CVD risk factor levels including glycated haemoglobin (HbA1c), blood pressure, cholesterol and body mass index (BMI). ${ }^{389} 10$ There is currently insufficient evidence to confirm whether PCC influences CVD risk factor levels among patients with diabetes, and the mechanism to explain any associations remains unclear. We hypothesise that the mechanism linking PCC to CVD risk factor levels is through patient health behaviours. ${ }^{11}$

The majority of diabetes care occurs in general practice where there is increasing pressure on GP consultation time. This is leading to a range of alternative chronic disease management strategies such as more routinised care, telehome care and remote monitoring, each of which may diminish PCC. ${ }^{12}{ }^{13}{ }^{14}$ With the need to optimise efficiency as well as effectiveness in diabetes care, it is increasingly important to assess the experience of PCC in improving disease risk. Evidence for the role of PCC in cost-effective diabetes care is needed to inform policy and has implications for the management of chronic disease more widely.

We aimed to quantify the association between the experience of PCC delivered by GPs and CVD risk factor levels at 1-year and 5-year follow-up in a wellcharacterised cohort of patients recently diagnosed with type 2 diabetes. To enable better understanding of the potential mechanisms underlying this association, we also examined associations between PCC and health behaviours.

\section{METHODS}

Study design

A detailed description of the ADDITION-Plus study design and rationale can be found elsewhere. ${ }^{15}$ In brief, ADDITION-Plus is a randomised-controlled trial among 34 general practices across East Anglia, UK. ADDITION-Plus examined the efficacy of a facilitator-led, theory-based behaviour change intervention for individuals with recently diagnosed type 2 diabetes. In total, 478 out of 1109 eligible individuals agreed to participate and were individually randomised to receive either intensive treatment alone $(n=239)$ or intensive treatment plus a facilitator-led individual behaviour change intervention $(n=239)$. The trial was not designed to influence patientpractitioner interactions and there were no differences in PCC measures, health behaviours, CVD risk factor levels between trial groups at 1 year, and no differences in the proceeding multivariate analyses between trial arms. Therefore, data for this analysis were pooled and treated as a cohort analysis. Participants in the trial were followed up for 5 years. All measurements were taken at baseline, 1-year and 5-year follow-up, except for objectively measured physical activity which was assessed at 1-year and 5-year follow-up, and PCC at 1-year follow-up. All participants gave written informed consent, and the study was approved by the Eastern Multi-Centre Research Ethics Committee (reference number 02/5/54). The trial is registered as ISRCTN99175498.

\section{Measurements and outcomes}

Self-reported health behaviour

Physical activity and dietary intake were assessed by selfreport using the validated EPIC Physical Activity Questionnaire (EPAQ-2) and semiquantitative food frequency questionnaire. ${ }^{16}$ Alcohol intake and smoking status (categorised as never smoked, ex-smoker or current smoker) were assessed by self-report questionnaire.

\section{Objective measures of health behaviour}

Physical activity was measured using a combined heart rate and movement sensor (Actiheart, CamNtech) worn for at least three consecutive days, as described previously. ${ }^{16}$ Resulting time-series data were summarised into physical activity energy expenditure (in $\mathrm{kJ} / \mathrm{kg} / \mathrm{day}$ ) - a measure of total physical activity, sedentary time (h/day) and moderate-to-vigorous physical activity (MVPA; min/ day) ${ }^{18}$ Plasma vitamin $\mathrm{C}$ levels (which offer an objective biomarker measure of fruit and vegetable intake) ${ }^{17}$ were measured using a Fluoroskan Ascent FL fluorometer. ${ }^{17} 19$

\section{Clinical and biochemical measures}

Clinical and biochemical measures were collected by trained staff following standardised protocols, as described elsewhere. ${ }^{15}$ Blood pressure was calculated as the mean of three measurements using an automatic sphygmomanometer. Body weight and height were measured in light clothing and without shoes using a scale (SECA) and a fixed rigid stadiometer, respectively. ${ }^{15}$ Venous blood samples were collected for analysis of lipid levels and HbA1c. Modelled 10-year cardiovascular risk was calculated using the UK Prospective Diabetes Study (UKPDS) risk engine (V.3.0).

\section{Patient-centred care}

PCC is a challenging concept to study or measure as there are multiple definitions and tools within the literature. At its core, PCC seeks to encompass the management of biophysical markers, alongside the human experience of disease. The consultation and relational empathy (CARE) measure is a holistic tool that attempts to capture PCC with a focus on the quality of consultations in terms of the 'human' aspects (empathic process of care). This is in the context of a doctor-patient interaction and from the patient's perspective. The CARE questionnaire is a measure that has been shown to be meaningful to patients, acceptable and easy to complete. It has been developed and extensively validated within the primary care setting, 
where the vast majority of type 2 diabetes care occurs in the UK. ${ }^{20}$ The CARE measure includes 10 questions based on a Likert scale ranging from 1 to 5 . A CARE summary measure was derived by summing the individual scores from the 10 individual questions, with a possible range of $10-50 .^{20}$ Participants completed a questionnaire on PCC experiences in relation to their GPs over the preceding year, in relation to diabetes care using the CARE measure at 1-year follow-up. ${ }^{21}$

\section{Statistical analysis}

Participant characteristics were summarised at baseline, 1-year and 5-year follow-up using means (SDs) or frequencies. Participants with incomplete data across time points were excluded from the analyses. Multivariate linear regression models were constructed to examine the prospective associations between baseline and 1-year follow-up, and between 1-year and 5-year follow-up between PCC measures and: (1) change in self-reported health behaviours; (2) change in objective health behaviours; (3) changes in biochemical and clinical measures; and (4) change in modelled 10-year cardiovascular risk. As physical activity was not measured objectively at baseline, this was examined cross-sectionally at 1 year. All models were adjusted, based on a priori reasoning, for age, sex, socioeconomic group, ethnicity, trial group, relevant medication use (ie, change in blood pressure, lipid or diabetes medications). Statistical analysis was performed using STATA/SE V.13.1 (STATA-Corp, College Station, Texas, USA). Statistical significance was set at $\mathrm{p}<0.05$.

\section{RESULTS}

\section{Participant characteristics}

Three hundred and ninety-six ADDITION-Plus participants had complete data and were included in these analyses. Participants had a mean (SD) age of 61 (6.9) years; the majority were Caucasian (96\%) and male (63\%; table 1). Seventy-four per cent of participants were in part-time or full-time employment and most continued in full-time education after the age of 16 years $(61 \%)$. Baseline mean (SD) HbAlc was 7.1 (1.4)\% (49.7 mmol/mol) (1.3). Change in clinical and biochemical variables at baseline, 1-year and 5-year follow-up is summarised in table 2. Mean BMI, waist circumference, HbAlc, blood pressure and cholesterol levels improved over the 5 years of follow-up. The mean (SD) CARE score was 39 (9.8) at 1-year follow-up. There were no significant differences in age, sex, ethnicity, employment status, social class, education, smoking status, blood pressure, lipid profile, waist circumference and 10-year modelled cardiovascular risk between participants with and without missing data for these analyses.

\section{Self-reported health behaviours}

Analysis of change from baseline to 1-year follow-up showed that participants reporting better experiences of
Table 1 Baseline characteristics in the ADDITION-Plus trial cohort $(n=396)$

\begin{tabular}{|c|c|}
\hline Variables & Mean $\pm S D$ \\
\hline \multicolumn{2}{|l|}{ Sociodemographic characteristics } \\
\hline Male sex, $\mathrm{n}(\%)$ & $252(63.1)$ \\
\hline Age at baseline (years) & $61(6.9)$ \\
\hline White ethnic origin, $n(\%)$ & $379(96)$ \\
\hline Employed, n (\%) & $296(74)$ \\
\hline \multicolumn{2}{|l|}{ Social class, $n(\%)$} \\
\hline High & $170(43.4)$ \\
\hline Manual & $173(44.3)$ \\
\hline Non-manual & $48(12.2)$ \\
\hline \multicolumn{2}{|l|}{ Education, n (\%) } \\
\hline Full-time education finished at $<16$ years & $150(38.4)$ \\
\hline Full-time education finished at $16-18$ years & $172(44.0)$ \\
\hline Full-time education finished at $>18$ years & $69(17.6)$ \\
\hline \multicolumn{2}{|l|}{ Medical history } \\
\hline History of angina, $\mathrm{n}(\%)$ & $47(10.7)$ \\
\hline History of hypertension, $\mathrm{n}(\%)$ & $175(40.2)$ \\
\hline $\begin{array}{l}\text { History of any cardiovascular disease } \\
\text { including AF }\end{array}$ & $50(11.6)$ \\
\hline History of myocardial infarct, $\mathrm{n}(\%)$ & $31(7.26)$ \\
\hline History of hypercholesterolaemia, n (\%) & $197(46.9)$ \\
\hline History of stroke, n (\%) & $13(3.1)$ \\
\hline \multicolumn{2}{|l|}{ Self-reported drug use } \\
\hline Any glucose-lowering drug, $\mathrm{n}(\%)$ & $126(32)$ \\
\hline Any antihypertensive drug, $\mathrm{n}(\%)$ & $280(71)$ \\
\hline Any cholesterol-lowering drug, $\mathrm{n}(\%)$ & $205(52)$ \\
\hline \multicolumn{2}{|l|}{ Self-reported lifestyle } \\
\hline $\begin{array}{l}\text { Physical activity energy expenditure, mean } \\
\text { (SD) (kJ/kg/day) }\end{array}$ & $29(7.4)$ \\
\hline \multicolumn{2}{|l|}{ Smoking status, n (\%) } \\
\hline Current smoker & $55(14)$ \\
\hline Ex-smoker & $196(49.7)$ \\
\hline Never smoker & $142(36)$ \\
\hline Alcohol per week (units), mean (SD) & 9 (13.9) \\
\hline
\end{tabular}

PCC were more likely to increase their self-reported physical activity by small amounts, including total activity energy expenditure ( $\beta$-coefficient: 0.080 MET h/day (95\% CI 0.017 to 0.143), MVPA ( $\beta$-coefficient: $5.328 \mathrm{~min} /$ day (95\% CI 0.796 to 9.859$)$ ), and reduce sedentary time ( $\beta$-coefficient: $-1.633 \mathrm{~min} /$ day $(95 \% \mathrm{CI}$ -2.897 to -0.368 ); table 3 ). We observed no clear associations between PCC and self-reported diet or alcohol intake. Over a longer follow-up from 1 to 5 years, there was no clear evidence that better experiences of PCC were associated with change in self-reported physical activity, diet or alcohol intake (table 3). We have not reported on change in smoking status as too few $(n=12)$ participants quit or started smoking to enable this to be examined.

\section{Objective health behaviours}

Over the first year of follow-up, there was no evidence that better experience of PCC were associated with objectively measured physical activity or diet (fruit and 
Table 2 Clinical variables of participants with complete data at all three time points

\begin{tabular}{lcccc}
\hline & N & Baseline & One-year follow-up & Five-year follow-up \\
\hline Clinical characteristics & & & & \\
BMI $\left(\mathrm{kg} / \mathrm{m}^{2}\right)$ & 383 & $32.4(5.6)$ & $31.8(5.1)$ & $31.9(5.4)$ \\
Waist circumference (cm) & 383 & $109.9(13.0)$ & $108.6(12.8)$ & $107.9(13.6)$ \\
HbA1c (\%), mean (SD) & 387 & $7.1(1.4)$ & $6.6(0.9)$ & $6.9(0.9)$ \\
Systolic blood pressure (mm Hg) & 396 & $136.8(19.7)$ & $130.2(17.7)$ & $132.2(16.4)$ \\
Diastolic blood pressure (mm Hg) & 396 & $80.7(10.6)$ & $76.4(9.5)$ & $73.9(9.8)$ \\
Total cholesterol (mmol/L) & 390 & $4.9(1.06)$ & $4.3(0.8)$ & $4.2(0.9)$ \\
HDL-cholesterol (mmol/L) & 390 & $1.2(0.3)$ & $1.2(0.3)$ & $1.3(0.3)$ \\
LDL-cholesterol (mmol/L) & 390 & $2.9(0.9)$ & $2.3(0.7)$ & $2.1(0.7)$ \\
\hline
\end{tabular}

Values are mean (SD).

BMI, body mass index; HbA1c, glycated haemoglobin; HDL, high-density lipoprotein; LDL, low-density lipoprotein.

vegetable intake measured with plasma vitamin $\mathrm{C}$ levels). Similarly, analysis of change between 1 and 5 years also demonstrated no associations between PCC and objectively measured diet or physical activity. These results are summarised in table 3 .

\section{Clinical and biochemical measures}

Analysis of change over the first year of follow-up demonstrated that participants with better experiences of PCC had marginally greater increases in high-density lipoprotein (HDL) cholesterol ( $\beta$-coefficient: $0.002 \mathrm{mmol} / \mathrm{L}(95 \%$ CI 0.001 to 0.004$)$ ) and decreases in both systolic blood pressure ( $\beta$-coefficient: $-0.561 \mathrm{~mm} \mathrm{Hg}(95 \% \mathrm{CI}-0.653$ to -0.468$)$ ) and diastolic blood pressure ( $\beta$-coefficient: $-0.565 \mathrm{~mm} \mathrm{Hg}(95 \%$ CI -0.654 to -0.476$))$. As shown in table 3 , there were no other associations between baseline and 1 year in clinical and biochemical measures. Over the longer 5-year follow-up, there were no associations between PCC and clinical or biochemical outcomes, except for waist circumference ( $\beta$-coefficient: $0.085 \mathrm{~cm}(95 \%$ CI 0.015 to 0.155$)$ ) which increased with higher PCC.

\section{DISCUSSION}

Better experience of PCC early after the diagnosis of type 2 diabetes was associated with a small, but not clinically meaningful change in self-reported physical activity, time spent sedentary, and improvements in HDL-cholesterol and blood pressure at 1-year. This was not reflected in the objective measures of physical activity. Over the longer term, we found no evidence to suggest that PCC was associated with changes in health behaviours or CVD risk factor levels. This study provides insufficient evidence that patients recently diagnosed with type 2 diabetes who have experiences of PCG are more likely to have lower cardiovascular risk factor levels via changes in patient health behaviours.

To the best of our knowledge, this is the first study to use objective measures of health behaviours alongside self-reported health behaviours to quantify the impact of experiences of PCC in a population with recently diagnosed type 2 diabetes. Furthermore, it includes a relatively long duration of follow-up of 5 years. We observed discrepancies in associations between PCC and self-reported, and objectively measured physical activity and diet. This highlights the potential bias associated with patient self-report questionnaires in previous studies. Other strengths include the use of a large number of GP surgeries which reflect average UK GP list sizes, diabetes prevalence, doctor or nurse whole time equivalent and patient experiences of diabetes care. Further, more than $50 \%$ of practices that were approached agreed to participate in the original study. The participant follow-up rate was also high at $95 \%$ at year follow-up and $83 \%$ at the 5-year follow-up. In relation to previous literature on PCC, this cohort study also includes a relatively large sample size. Additional strengths include our measure of PCC; while some previous studies have used non-specific and non-validated patient satisfaction questionnaires as a marker of PCC, we used the validated CARE measure.8 102122 The validity and reliability of the CARE measure has been extensively demonstrated, and applied in over 3000 general practice consultations in areas of high and low deprivation and across multiple health conditions.

A number of limitations of our study also warrant discussion. We measured PCC at a single time point at 1-year follow-up which may explain differences between 1-year and 5-year results. Further, because doctor-patient relationships are dynamic and are established or changed over time, ${ }^{23}$ we were not able to examine how changes in experiences of PCG might affect health behaviours and CVD risk factor levels. The majority of participants were Caucasian males with high levels of education and employment, thereby limiting the generalisability of our findings as experiences of PCC and diabetes care may differ in a more ethnically diverse or socially deprived populations. The majority of participants reported high CARE scores which, due to homogeneity, will likely have reduced our ability to identify associations with health outcomes. Finally, we also conducted a number of hypothesis tests and as a result we cannot exclude the role of chance as a plausible explanation for our findings.

Previous studies examining the association of interventions to alter PCC and CVD risk factor levels in type 2 
Table 3 Linear associations between patient-centred care and outcomes at 1- and 5-year follow-up in ADDITION-Plus cohort

\begin{tabular}{|c|c|c|c|c|c|c|c|c|c|}
\hline \multirow[b]{2}{*}{ Variable } & \multirow[b]{2}{*}{$\mathbf{N}$} & \multicolumn{4}{|c|}{ Changes from 0 to 1 years of follow-up } & \multicolumn{4}{|c|}{ Changes from 1 to 5 years of follow-up } \\
\hline & & Coefficient & & $95 \% \mathrm{Cl}$ & p Value & Coefficient & & $95 \% \mathrm{Cl}$ & p Value \\
\hline \multicolumn{10}{|l|}{ Self-reported measures } \\
\hline Total activity energy expenditure (MET h/day) & 371 & 0.080 & 0.017 & 0.143 & 0.01 & -0.037 & -0.318 & 0.243 & 0.79 \\
\hline Sedentary time (min/day) & 371 & -1.633 & -2.897 & -0.368 & 0.01 & 0.014 & -0.010 & 0.037 & 0.25 \\
\hline Moderate-to-vigorous physical activity (min/day) & 371 & 5.328 & 0.796 & 9.859 & 0.01 & -0.241 & -0.880 & 0.400 & 0.46 \\
\hline Energy intake (kJ/day) & 371 & 0.920 & -3.960 & 5.810 & 0.71 & 0.012 & 0.001 & 0.001 & 0.51 \\
\hline Alcohol per week (units) & 371 & 0.022 & -0.037 & 0.081 & 0.47 & -0.022 & -0.085 & 0.041 & 0.49 \\
\hline \multicolumn{10}{|l|}{ Objectively measured health behaviours } \\
\hline Physical activity energy expenditure $(\mathrm{kJ} / \mathrm{kg} / \text { day })^{\star}$ & 308 & -0.001 & -0.166 & 0.164 & 0.99 & -0.014 & 0.850 & -0.100 & 0.08 \\
\hline Plasma vitamin $C(\mu \mathrm{mol} / \mathrm{L})$ & 303 & -0.231 & -0.462 & $<0.001$ & 0.05 & -0.040 & -0.100 & 0.020 & 0.17 \\
\hline \multicolumn{10}{|l|}{ Clinical and biochemical measures } \\
\hline $\mathrm{HbA} 1 \mathrm{c}(\%) \dagger$ & 387 & -0.006 & -0.015 & 0.004 & 0.23 & 0.004 & -0.005 & 0.013 & 0.39 \\
\hline Systolic blood pressure $(\mathrm{mm} \mathrm{Hg}) \ddagger$ & 396 & -0.561 & -0.653 & -0.468 & 0.01 & 0.107 & -0.053 & 0.267 & 0.19 \\
\hline Diastolic blood pressure $(\mathrm{mm} \mathrm{Hg}) \ddagger$ & 396 & -0.565 & -0.654 & -0.476 & 0.01 & 0.064 & -0.031 & 0.159 & 0.19 \\
\hline Total cholesterol $(\mathrm{mmol} / \mathrm{L}) \S$ & 390 & 0.002 & -0.006 & 0.011 & 0.58 & 0.001 & -0.008 & 0.010 & 0.83 \\
\hline HDL-cholesterol $(\mathrm{mmol} / \mathrm{L}) \S$ & 390 & 0.002 & 0.001 & 0.004 & 0.03 & -0.002 & -0.004 & 0.001 & 0.17 \\
\hline LDL-cholesterol $(\mathrm{mmol} / \mathrm{L}) \S$ & 390 & 0.007 & 0.001 & 0.014 & 0.07 & 0.001 & -0.007 & 0.007 & 0.99 \\
\hline Waist circumference $(\mathrm{cm})$ & 383 & -0.060 & -0.120 & 0.011 & 0.07 & 0.085 & 0.015 & 0.155 & 0.02 \\
\hline $\mathrm{BMI}\left(\mathrm{kg} / \mathrm{m}^{2}\right)$ & 383 & -0.010 & -0.031 & 0.006 & 0.19 & 0.013 & -0.010 & 0.036 & 0.27 \\
\hline Modelled UKPDS 10-year cardiovascular riskף & 390 & 0.001 & -0.001 & $<0.001$ & 0.54 & 0.001 & -0.001 & 0.001 & 0.51 \\
\hline
\end{tabular}

${ }^{*}$ Measured at 1-year only.

†Adjusted for sex, age, ethnicity, social class and hypoglycaemic medication.

$\ddagger$ Adjusted for sex, age, ethnicity, social class and antihypertensive medication.

§Adjusted for sex, age, ethnicity, social class and lipid-lowering therapy.

IAdjusted for sex, age, ethnicity, social class, lipid-lowering therapy, antihypertensive and hypoglycaemic medication.

BMI, body mass index; HbA1c, glycated haemoglobin; HDL, high-density lipoprotein; LDL, low-density lipoprotein; UKPDS, UK Prospective Diabetes Study. 
diabetes have reported mixed results. This may be related to the fact that PCC is a broad term with multiple descriptions and measures, and therefore a high level of heterogeneity exists between studies on this subject. ${ }^{24}$ We found positive associations, albeit clinically not meaningful, between PCC and self-reported physical activity level, blood pressure and HDL-cholesterol, that is - people with better PCC experiences reported being more physically active and had higher HDL-cholesterol levels and lower blood pressures at 1-year. This is consistent with some previous observational and trial data, ${ }^{22} 25262728$ except our study includes objective measures and therefore overcomes some of the limitations associated with previous self-reported data. Several studies have also reported inverse associations between PCC and non HDL-cholesterol, ${ }^{25}{ }_{28} 22$ BMI, HbAlc $^{29} \quad 3031$ and cardiovascular risk. ${ }^{32}$ We did not observe such associations at 1-year or 5-year follow-up. ${ }^{102530}$ Differences may have been because our study was underpowered to detect these changes, or might be related to our measure of PCC. Our study is the first to use the CARE measure as a specific marker of PCC focusing on empathy in patients recently diagnosed with type 2 diabetes that were followed up over a 5 -year period. ${ }^{20}$ These differences, as well as the potential role of chance, may also explain the positive, albeit small, unexpected association between PCC and waist circumference.

Further, baseline measures also vary across studies which may explain differences in findings. For example, mean $\mathrm{HbAlc}$ in participants in our cohort at baseline was $7.1 \%$. A recent large study in type 2 diabetes within secondary care demonstrated significant reductions in HbAlc following a PCC intervention. ${ }^{31}$ This study suggested that a PCC approach may be most effective in improving glycaemic control when baseline HbAlc is over $8.5 \%$, and reported modest effects in patients with an $\mathrm{HbAlc}$ below $7 \%$. Previous studies in primary care have similarly demonstrated a greater effect of PCC when baseline HbAlc was high. ${ }^{22} 293033$ We therefore carried out a post hoc analysis including only participants with HbAlc over $8.5 \%$ at baseline, and found stronger associations between PCC, physical activity, and HbA1c, non-HDL-cholesterol and BMI, but these associations did not reach statistical significance, likely owing to the reduction is sample size and therefore statistical power.

The literature is bedeviled with lack of clear definition and measures of PCC in terms of interactions with health professionals. ${ }^{24} 34{ }^{35}$ More frequent use of standardised and validated measures of PCC in future research will reduce heterogeneity and allow comparison between studies on PCC. Further, most studies use selfreported measures of health behaviours which are prone to reporting error and bias, as demonstrated by the lack of consistency between our subjective and objective assessments. Social desirability bias may be one explanation for the higher levels of self-reported health behaviours compared with objective health behaviours observed in our study. This highlights the need for future research to include objective measures of outcomes. Further, we could not exclude reverse causality as a potential explanation for this and previous findings. Future well-conducted trials alongside qualitative work are essential to explore the mechanism linking PCC, health behaviours and outcomes. Also, we found stronger associations between PCC among people with poor glycaemic control, albeit not significant. This has been suggested previously, ${ }^{31}$ and future research will need to stratify disease severity and patient groups to further examine the role of PCC in these particular groups of patients.

Current National Health Service (NHS) healthcare policy emphasises the importance of 'making every contact count', and highlights the role that GPs have to play in modifying health behaviours and secondary disease risk. Our study provides insufficient evidence to exclude that PCC is associated with improvements in health-related behaviours or CVD risk factor levels in the first 5 years following diagnosis. Although PCC is preferred by our patients and often considered a moral imperative or the 'right thing' for clinicians to do, it is important to adequately balance PCC against evidencebased disease management strategies in type 2 diabetes. $^{36}$

\section{Author affiliations}

${ }^{1}$ Primary Care Unit, Department of Public Health and Primary Care, University of Cambridge, Cambridge, UK

${ }^{2}$ MRC Epidemiology Unit, University of Cambridge School of Clinical Medicine, Institute of Metabolic Science, Cambridge Biomedical Campus, Cambridge, UK

Acknowledgements The authors would like to thank Professor Stewart Mercer for reviewing an early draft of this paper and for the helpful guidance on the use of the CARE measure. They are grateful to all participants and the practices teams for taking part in the ADDITION-Plus trial-Arbury Road Surgery, Ashwell Surgery, Birchwood Surgery, Bottisham Medical Practice, Brookfields/Cherry Hinton, Buckden Surgery, Clarkson Surgery, Cornerstone Practice, Cornford House Surgery, Cottenham Surgery, Dr Eaton \& Partners (Saffron Walden), George Clare Surgery, Great Staughton Surgery, Haddenham Surgery, Hilton House Surgery, Manea Surgery, Milton Surgery, New Roysia Surgery, Orchard House Surgery, Orton Medical Practice, Lensfield Road Surgery, Parkhall Road Surgery, Park Medical Centre, Petersfield Medical Practice, Riverside Practice, Rookery Medical Centre, Rosalind Franklin House, South Street Surgery, St Mary's Surgery, Thaxted Surgery, The Old Exchange, The Spinney Surgery, The Surgery (Over), and Woolpit Surgery. They also thank the Cambridge University Hospitals NHS Foundation Trust Department of Clinical Biochemistry and the NIHR Cambridge Biomedical Research Centre, Core Biochemical Assay Laboratory for carrying out the biochemical assays.

Collaborators The ADDITION- Plus team also includes: From the Medical Research Council Epidemiology Unit, Cambridge: Ruhul Amin, Gisela Baker, Mark Betts, Adam Dickinson, Justin Basile Echouffo Tcheugui, Ulf Ekelund, Francis Finucane, Stefanie Mayle, Joanna Mitchell, Paul Roberts, Lincoln Sargeant, Matt Sims, Nick Wareham, Fiona Whittle and the Field Epidemiology, Data Management, IT, Physical Activity Technical and Study Coordination teams. From the Primary Care Unit, University of Cambridge: Judith Argles, Rebecca Bale, Roslyn Barling, Sue Boase, James Brimicombe, Ryan Butler, Tom Fanshawe, Philippa Gash, Julie Grant, Wendy Hardeman, Imogen Hobbis, Ann Louise Kinmonth, Tom McGonigle, Nicola Popplewell, 
Jenny Smith, Megan Smith, Stephen Sutton, Fiona Whittle and Kate Williams. The Primary Care Unit, University of Cambridge (GP) and the Medical Research Council Epidemiology Unit in Cambridge (MRC) jointly coordinated the baseline and 1-year follow-up phases of the study.

Contributors HD-M conducted the analysis of the data, wrote the analysis plan, drafted and revised the paper. AJMC conducted analysis and revised the paper. RKS revised the paper. SJG and ALK initiated the project, designed the ADDITION trial and data collection tools, implemented the trial, monitored data collection for the whole trial, and revised the paper. SJG is guarantor.

Funding The trial is supported by the Medical Research Council (grant reference no: G0001164), the Wellcome Trust (grant reference no: G061895), Diabetes UK and National Health Service R\&D support funding. SJG is a member of the National Institute for Health Research (NIHR) School for Primary Care Research. The General Practice and Primary Care Research Unit was supported by NIHR Research funds.

Disclaimer The views expressed in this publication are those of the authors and not necessarily those of the Department of Health.

Competing interests None declared

Ethics approval Ethics committee approval was obtained from the Eastern Multi-Centre Research Ethics Committee (reference number: 02/5/54). The trial was registered as ISRCTN 99175498

Provenance and peer review Not commissioned; externally peer reviewed.

Data sharing statement No additional data are available.

Open Access This is an Open Access article distributed in accordance with the terms of the Creative Commons Attribution (CC BY 4.0) license, which permits others to distribute, remix, adapt and build upon this work, for commercial use, provided the original work is properly cited. See: http:// creativecommons.org/licenses/by/4.0/

\section{REFERENCES}

1. Zimmet P, Alberti KG, Shaw J. Global and societal implications of the diabetes epidemic. Nature 2001;414:782-7.

2. Toop L. Primary care: core values patient centred primary care. BMJ 1998;316:1882-3.

3. Griffin SJ, Kinmonth A-L, Veltman MWM, et al. Effect on health-related outcomes of interventions to alter the interaction between patients and practitioners: a systematic review of trials. Ann Fam Med 2004;2:595-608.

4. NHS Yorskhire and Humber. 2012. Make every contact count. http:// www.healthyambitions.co.uk/Uploads/BetterForLess/08 BETTER FOR LESS every contact counts.pdf (accessed 24 Nov 2014).

5. Scarborough $\mathrm{P}$, Bhatnagar $\mathrm{P}$, Wickramasinghe $\mathrm{KK}$, et al. The economic burden of ill health due to diet, physical inactivity, smoking, alcohol and obesity in the UK: an update to 2006-07 NHS costs. J Public Health (Oxf) 2011;33:527-35.

6. NHS England 2014. Strategic and Operational Planning 2014 to 20192. Prevention and health promotion. http://www.england.nhs.uk ourwork/sop/red-prem-mort/php/ (accessed 5 Dec 2014).

7. Gensichen J, Von Korff M, Rutter CM, et al. Physician support for diabetes patients and clinical outcomes. BMC Public Health 2009;9:367.

8. Kelley JM, Kraft-Todd G, Schapira L, et al. The influence of the patient-clinician relationship on healthcare outcomes: a systematic review and meta-analysis of randomized controlled trials. PLOS ONE 2014;9:e94207

9. van Dam HA, van der Horst $F$, van den Borne $B$, et al. Providerpatient interaction in diabetes care: effects on patient self-care and outcomes. Patient Educ Couns 2003;51:17-28.

10. Kinmonth AL, Woodcock A, Griffin S, et al. Randomised controlled trial of patient centred care of diabetes in general practice: impact on current wellbeing and future disease risk. The Diabetes Care From Diagnosis Research Team. BMJ 1998;317:1202-8.

11. Khaw K-T, Wareham N, Bingham S, et al. Combined impact of health behaviours and mortality in men and women: the EPIC-Norfolk prospective population study. PLoS Med 2008;5:e12.

12. Walsh T, Barr PJ, Thompson R, et al. Undetermined impact of patient decision support interventions on healthcare costs and savings: systematic review. BMJ 2014;348:g188.
13. Schroter S, Jenkins RD, Playle RA, et al. Evaluation of an online diabetes needs assessment tool (DNAT) for health professionals: a randomised controlled trial. Trials 2009;10:63.

14. Henderson C, Knapp M, Fernández JL, et al. NS. Cost effectiveness of telehealth for patients with long term conditions (Whole Systems Demonstrator telehealth questionnaire study): nested economic evaluation in a pragmatic, cluster randomised controlled trial. BMJ 2013;346:f1035

15. Griffin SJ, Simmons RK, Williams KM, et al. Protocol for the ADDITION-Plus study: a randomised controlled trial of an individually-tailored behaviour change intervention among people with recently diagnosed type 2 diabetes under intensive UK general practice care. BMC Public Health 2011;11:211.

16. Brage S, Brage N, Franks PW, et al. Reliability and validity of the combined heart rate and movement sensor Actiheart. Eur J Clin Nutr 2005;59:561-70.

17. Bingham SA, Gill C, Welch A, et al. Validation of dietary assessment methods in the UK arm of EPIC using weighed records, and 24-hour urinary nitrogen and potassium and serum vitamin $C$ and carotenoids as biomarkers. Int J Epidemiol 1997;26(Suppl 1): S137-51.

18. Henry C. Basal metabolic rate studies in humans: measurement and development of new equations. Public Health Nutr 2007;8:1133-52.

19. Drewnowski A, Rock CL, Henderson SA, et al. Serum beta-carotene and vitamin $\mathrm{C}$ as biomarkers of vegetable and fruit intakes in a community-based sample of French adults. Am J Clin Nutr 1997;65:1796-802.

20. Mercer SW, Maxwell M, Heaney D, et al. The consultation and relational empathy (CARE) measure: development and preliminary validation and reliability of an empathy-based consultation process measure. Fam Pract 2004;21:699-705.

21. Mercer SW, McConnachie A, Maxwell M, et al. Relevance and practical use of the Consultation and Relational Empathy (CARE) Measure in general practice. Fam Pract 2005;22:328-34.

22. Olivarius NF, Beck-Nielsen $\mathrm{H}$, Andreasen $\mathrm{AH}$, et al. Randomised controlled trial of structured personal care of type 2 diabetes mellitus. BMJ 2001;323:970-5.

23. Stewart M, Brown JB, Weston WW, et al. Patient-centred medicine: transforming the clinical method. 2nd edn. UK: Radcliffe Medical Press, 2003

24. Stewart M. Towards a global definition of patient centred care. BMJ 2001;322:444-5.

25. Deakin TA, Cade JE, Williams $R$, et al. Structured patient education the diabetes X-PERT Programme makes a difference. Diabet Med 2006;23:944-54.

26. Hardcastle S, Taylor A, Bailey $\mathrm{M}$, et al. A randomised controlled trial on the effectiveness of a primary health care based counselling intervention on physical activity, diet and CHD risk factors. Patient Educ Couns 2008;70:31-9.

27. Boulware LE, Daumit GL, Frick KD, et al. An evidence-based review of patient-centered behavioral interventions for hypertension. $A m ~ J$ Prev Med 2001;21:221-32.

28. Piatt G a., Anderson RM, Brooks MM, et al. 3-year follow-up of clinical and behavioral improvements following a multifaceted diabetes care intervention: results of a randomized controlled trial. Diabetes Educ 2010;36:301-9.

29. Piette JD, Weinberger M, Mcphee SJ, et al. The effect of automated calls with telephone nurse on patient-centered outcomes of diabetes care follow-up. 2009;38:218-30.

30. Ralston JD, Hirsch IB, Hoath J, et al. Web-based collaborative care for type 2 diabetes: a pilot randomized trial. Diabetes Care 2009;32:234-9.

31. Slingerland AS, Herman WH, Redekop WK, et al. Stratified patient-centered care in type 2 diabetes: a cluster-randomized, controlled clinical trial of effectiveness and cost-effectiveness. Diabetes Care 2013;36:3054-61.

32. Davies MJ, Heller S, Skinner TC, et al. Effectiveness of the diabetes education and self management for ongoing and newly diagnosed (DESMOND) programme for people with newly diagnosed type 2 diabetes: cluster randomised controlled trial. BMJ 2008;336:491-5.

33. Anderson RM, Funnell MM, Butler PM, et al. Patient empowerment. Results of a randomized controlled trial. Diabetes Care 1995;18:943-9.

34. Mead N, Bower P. Patient-centredness: a conceptual framework and review of the empirical literature. Soc Sci Med 2000;51:1087-110.

35. Hudon C, Fortin M, Haggerty JL, et al. Measuring patients' perceptions of patient-centered care: a systematic review of tools for family medicine. Ann Fam Med 2011;9:155-64.

36. Little P. Preferences of patients for patient centred approach to consultation in primary care: observational study. BMJ 2001;322:468-468. 